B (72\%) and Child C (75\%) patients in comparison to child a patients $(52 \%)(\mathrm{p}=0.013)$ (figure 1$)$.

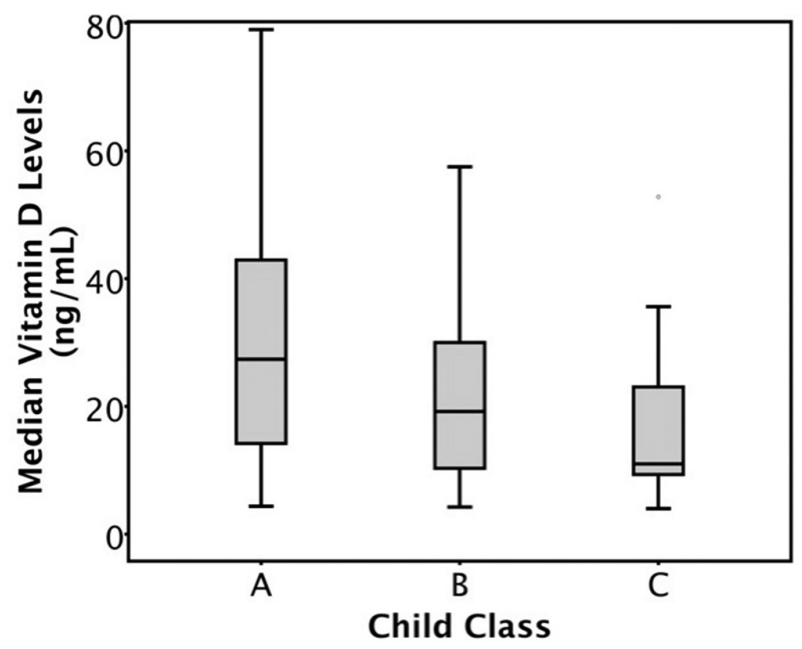

Abstract IDDF2018-ABS-0189 Figure 1 Median vitamin d levels according to child pugh status
Conclusions 25-Hydroxy Vitamin D deficiency was present in $61 \%$ and diabetes mellitus co-existed in $21 \%$ of HCV patients at presentation. Diabetes and low levels of $25-\mathrm{OH}$ vitamin $\mathrm{D}$ at presentation were associated with higher incidence of cirrhosis and HCC. We believe that improving diabetes control and correcting vitamin D deficiency can improve outcomes in hepatitis $\mathrm{C}$ related liver disease.

\section{IDDF2018-ABS-0190 PREDICTORS OF SHORT-TERM MORTALITY IN SEVERE ALCOHOLIC HEPATITIS AND USE OF STEROIDS IN CLINICAL PRACTICE: AN EXPERIENCE OUTSIDE CLINICAL TRIAL FROM INDIA}

Ravi Daswani*, Ashish Kumar, Shivam Khare, A Shrihari, Praveen Sharma, Naresh Bansal, Vikas Singla, Anil Arora. Sir Gangaram Hospital, New Delhi, India

\subsection{6/gutjnl-2018-IDDFabstracts.230}

Background Severe $\mathrm{AH}$ (SAH) is the most florid presentation of alcohol-related liver disease with high short-term mortality. Steroids have remained the mainstay of therapy despite the controversy. The aim of the present study was to assess the risk factors and compare the severity scores predicting 90 day

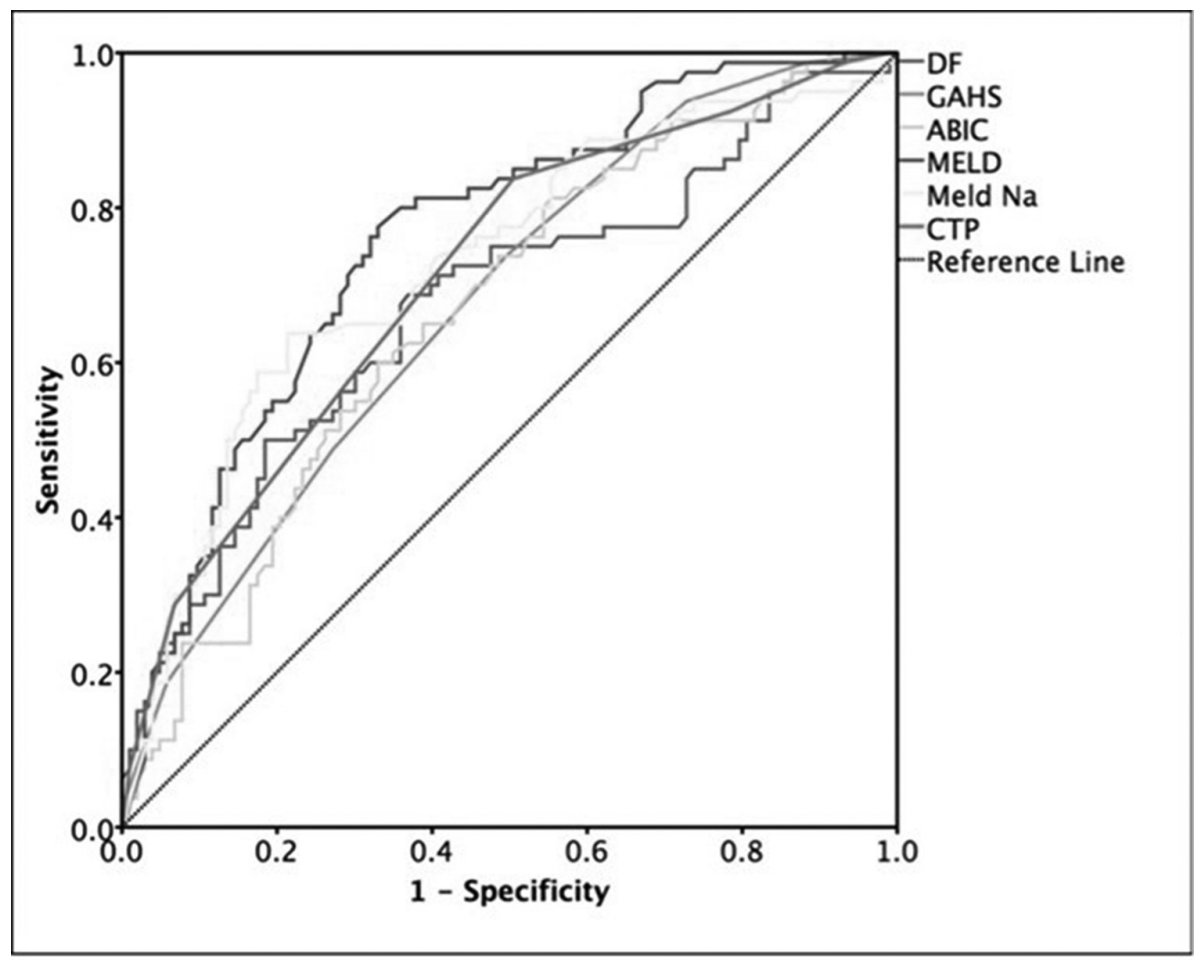

\begin{tabular}{|l|c|c|c|c|c|}
\hline \multirow{2}{*}{ Score } & \multirow{2}{*}{ AUC } & Std error & \multirow{2}{*}{ P value } & \multicolumn{2}{|c|}{$95 \% \mathrm{Cl}$} \\
\cline { 5 - 6 } & & & & Lower & Upper \\
\hline DF & 0.675 & 0.041 & $<0.001$ & 0.595 & 0.755 \\
\hline GAHS & 0.675 & 0.039 & $<0.001$ & 0.599 & 0.752 \\
\hline ABIC & 0.666 & 0.040 & $<0.001$ & 0.588 & 0.744 \\
\hline MELD & 0.761 & 0.035 & $<0.001$ & 0.691 & 0.830 \\
\hline Meld Na & 0.728 & 0.038 & $<0.001$ & 0.653 & 0.803 \\
\hline CTP & 0.713 & 0.038 & $<0.001$ & 0.638 & 0.788 \\
\hline
\end{tabular}


mortality along with the feasibility of steroids in this sick subset of patients.

Methods All patients with SAH (defined as Discriminant Function $\geq 32$ ) admitted from January 2015 to February 2017 at our Institute were prospectively enrolled. All patients were given standard treatment according to various guidelines, and their 90 day mortality was determined. Various hematologic, biochemical factors and severity scores were compared between survivors and patients who died.

Results A total of 183 patients (98\% males, median age 41 years [range 20-70 years]) were included. The median MELD was 26 (15-40). Ascites was present in $83 \%$ and hepatic encephalopathy in 38\%. Only $21(12 \%)$ could be offered steroid therapy, due to contraindications in the remaining. By 90 days, only $103(56 \%)$ patients survived while 80 (44\%) patients had died. All patients died due to progressive liver failure and its complications. On multivariate analysis presence of ascites, hepatic encephalopathy, high bilirubin, low albumin, high creatinine, high INR, and low potassium independently predicted 90 day mortality. All the scores performed significantly in predicting 90 day mortality with no statistically significant difference between them. MELD score had a maximum area under the curve 0.76 for 90 day mortality (table 1 and figure1). A combination of Child class and presence of acute kidney injury (creatinine $\geq 1.35$ ) was good in predicting 90 day mortality.

\begin{tabular}{lcccccc}
\multicolumn{6}{l}{ Abstract IDDF2018-ABS-0190 Table 1} \\
\hline Score & AUC & $\begin{array}{c}\text { Std } \\
\text { error }\end{array}$ & P value & \multicolumn{2}{c}{$95 \% \mathrm{Cl}$} \\
\cline { 5 - 7 } & & & & Lower & Upper \\
\hline DF & 0.675 & 0.041 & $<0.001$ & 0.595 & 0.755 \\
GAHS & 0.675 & 0.039 & $<0.001$ & 0.599 & 0.752 \\
ABIC & 0.666 & 0.040 & $<0.001$ & 0.588 & 0.744 \\
MELD & 0.761 & 0.035 & $<0.001$ & 0.691 & 0.830 \\
Meld & 0.728 & 0.038 & $<0.001$ & 0.653 & 0.803 \\
Na & & & & & \\
CTP & 0.713 & 0.038 & $<0.001$ & 0.638 & 0.788 \\
\hline
\end{tabular}

Conclusions In India, SAH is characterised by a median MELD score of 26 and has a 90 day mortality of 44\%. Most Indian

\section{A}

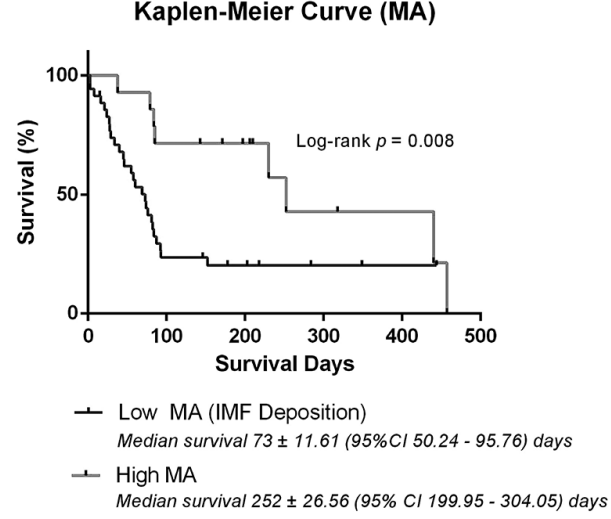

patients are not eligible to receive corticosteroids. Presence of Child $C$ status and high serum creatinine value $(\geq 1.35 \mathrm{mg} / \mathrm{dL})$ accurately predicts mortality and is an easy and effective bedside tool to prognosticate the patients at admission. Newer treatment options need to be explored for these patients.

\begin{tabular}{l|l}
\hline IDDF2018-ABS-0195 & REDUCED MUSCLE (RADIO) DENSITY AND \\
& BARCELONA CLINIC LIVER CANCER (BCLC) \\
& STAGING ARE INDEPENDENTLY SURVIVAL \\
& PROGNOSTIC OF INDONESIA \\
& HEPATOCELULAR CARCINOMA (HCC) \\
& PATIENTS
\end{tabular}

Yan Mardian*, ${ }^{2}$ Yoshihiko Yano, ${ }^{3}$ Neneng Ratnasari, ${ }^{4}$ Lina Choridah, ${ }^{3}$ Fahmi Indrarti, ${ }^{3}$ Putut Bayupurnama, ${ }^{3}$ Catharina Triwikatwani, ${ }^{4}$ Nurhuda Hendra Setyawan, ${ }^{5}$ Yoshitake Hayashi. 'Division of Infectious Disease Pathology, Department of Microbiology and Infectious Disease, Kobe University Graduate School of Medicine, Kobe, Japan; ${ }^{2}$ Department of Gastroenterology, Kobe University Graduate School of Medicine, Kobe, Japan; ${ }^{3}$ Division of Gastroenterohepatology, Department of Internal Medicine, Dr. Sardjito Hospital, Faculty of Medicine, Gadjah Mada University, Yogyakarta, Indonesia; ${ }^{4}$ Department of Radiology, Dr. Sardjito Hospital, Faculty of Medicine, Gadjah Mada University, Yogyakarta, Indonesia; ${ }^{5}$ Division of Molecular Medicine and Medical Genetics, Department of Pathology, Kobe University Graduate School of Medicine, Kobe, Japan

\subsection{6/gutjnl-2018-IDDFabstracts.231}

Background Body composition components (Sarcopenia and intramuscular fat [IMF] deposition) were proven as risk factors predicting poor survival among HCC patients in the Japanese population. Since HCC characteristics were peculiar in Indonesia: advanced-stage at presentation; early-age onset; and HBV endemicity; our objective was to demonstrate key determinants of HCC prognosis in Indonesia.

Methods Skeletal muscle index (SMI) and mean muscle attenuation (MA) were measured from transverse Computed Tomography (CT) images at the third lumbar vertebra (L3) in a prospective cohort of 49 Indonesia patients with different stages of HCC. Images were analysed using SliceOmatic V5.0 (Tomovision, QC Canada), which enables specific tissue demarcation using Hounsfield unit (HU) thresholds. Clinical, laboratory and body composition assessments are comprehensively analysed using Kaplan-Meier procedure and Cox's

B

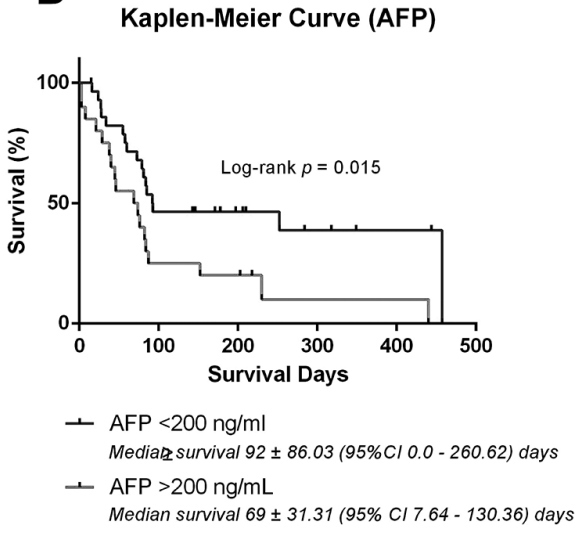

deposition and normal muscle attenuation ma b Kaplan meier curve indicating the survival of patients with alpha fetoprot) 